\title{
Order from disorder with intrinsically disordered peptide amphiphiles
}

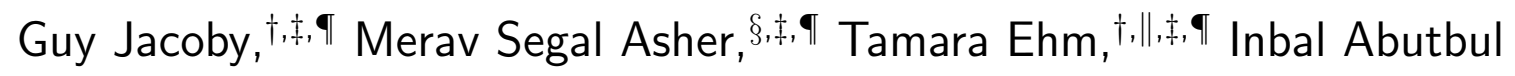 \\ Ionita, ${ }^{\perp}$ Hila Shinar, ${ }^{\dagger, \ddagger}, \mathbb{\uparrow}$ Salome Azoulay-Ginsburg, ${ }^{\S}$ Dganit Danino, ${ }^{\perp, \#}$ Michael
}

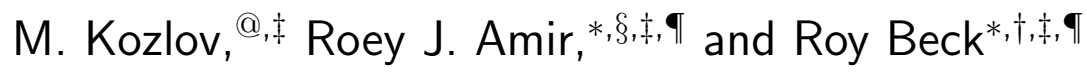

$\dagger$ Raymond $\mathcal{E}$ Beverly Sackler School of Physics $\mathcal{E}$ Astronomy, Tel Aviv University, Tel Aviv 6997801, Israel

$\ddagger$ The Center for Physics and Chemistry of Living Systems, Tel Aviv University, Israel

\The Center for NanoTechnology and NanoScience, Tel Aviv Univeristy, Israel $\S$ Raymond 83 Beverly Sackler School of Chemistry, Tel Aviv University, Tel Aviv 6997801, Israel

\| Faculty of Physics and Center for NanoScience, Ludwig-Maximilians-Universität, München, Germany

$\perp$ CryoEM Laboratory of Soft Matter, Faculty of Biotechnology and Food Engineering, Technion-Israel Institute of Technology, Haifa 3200003, Israel \#Guangdong-Technion Israel Institute of Technology, Shantou, Guangdong Province, 515063 China

@Sackler School of Medicine, Tel Aviv University, Tel Aviv 6997801, Israel

E-mail: amirroey@tauex.tau.ac.il; roy@tauex.tau.ac.il

\begin{abstract}
Amphiphilic molecules and their self-assembled structures have long been the target of extensive research due to their potential applications in fields ranging from materials
\end{abstract}


design to biomedical and cosmetic applications. Increasing demands for functional complexity have been met with challenges in biochemical engineering, driving researchers to innovate in the design of new amphiphiles. An emerging class of molecules, namely, peptide amphiphiles, combines key advantages and circumvents some of the disadvantages of conventional phospholipids and block-copolymers. Herein, we present new peptide amphiphiles comprised of an intrinsically disordered peptide conjugated to two variants of hydrophobic dendritic domains. These molecules termed intrinsically disordered peptide amphiphiles (IDPA), exhibit a sharp pH-induced micellar phasetransition from low-dispersity spheres to extremely elongated worm-like micelles. We present an experimental characterization of the transition and propose a theoretical model to describe the pH-response. We also present the potential of the shape transition to serve as a mechanism for the design of a cargo hold-and-release application. Such amphiphilic systems demonstrate the power of tailoring the interactions between disordered peptides for various stimuli-responsive biomedical applications.

\section{Introduction}

Self-assembly of amphiphilic molecules holds great interest from a fundamental scientific point of view, as well as for their potential for creating nanocarriers for various applications, ranging from drugs and nucleic acids in medicine to fragrances and other small chemicals in the food and cosmetics industries. ${ }^{1}$ The functionality of nanocarriers' design will depend on many factors, including the mechanism for cargo hold-and-release, bio-compatibility, uniformity, and tunability, all of which present challenging obstacles in engineering efficient nanocarriers. Natural lipids and synthetic block-copolymers are two of the most widely used amphiphiles in designing such systems, each prevailing due to its specific advantages ${ }^{2-12}$

An emerging class of synthetic amphiphiles, namely, peptide amphiphiles (PA), is designed to self-assemble into functional structures by building upon the advantageous characteristics of lipids and block-copolymers. ${ }^{13-15}$ The molecules' hydrophilic domain, usually a 
bio-inspired peptide engineered to fulfill one or more active roles, is chemically conjugated to a hydrophobic tail group, usually single or double chain fatty acids like those found in lipids. Due to their highly flexible design scheme, PA self-assemblies can act as organic scaffolds for bone-like mineralization, ${ }^{16}$ anisotropic actuators mimicking skeletal muscle, ${ }^{17}$ produce new versatile soft materials, ${ }^{18}$ and enhance neural progenitor cell differentiation into neurons. ${ }^{19}$ PA can contain spacers or linkers in their design, connecting the hydrophobic tails to the functional hydrophilic domain, ${ }^{20,21}$ or conjugating to an "external" functional group, such as an MRI contrast agent. ${ }^{22}$ Comprehensive research is done to understand the properties and roles of the different molecular domains and their contribution to the specific self-assembled mesophase. ${ }^{23,24}$ In many cases, the bio-inspired peptides are designed or derived from proteins with some degree of secondary structure. ${ }^{25}$ Nonetheless, PA hydrophilic domains are not exclusively bio-inspired and can be made of any polypeptide sequence, including intrinsically disordered (i.e., unfolded) peptide sequences.

An increasingly large number of proteins have been found to lack a fixed or ordered structure. ${ }^{26}$ As such, these proteins have been termed intrinsically disordered proteins (IDPs). A more operational definition of an IDP is a protein that does not possess only a single functional conformation, but rather it can fold into an ensemble of functional conformations depending on the setting. This structural plasticity is, in many cases, the result of relatively weaker and transient interactions between proteins segments. ${ }^{27-30}$ The nature of their interactions makes IDPs better suited for specific roles than structured proteins as they can interact with multiple partners or retain in liquid condensed phase. ${ }^{31,32}$

Envisioning systems with intricate and precise self-organization potential, as sought after in applications, requires the fabrication and processing of unique nanostructures. A primary limitation of current block-copolymer synthesis is the lack of general methods for producing precise chain structure (i.e., sequence control) to facilitate multiple desired functions. Natural lipids also have limited functionality due to their relatively small hydrophilic domain. In contrast, intrinsically disordered peptide amphiphiles (IDPAs) benefit from the 
use of relatively short sequences that are easier to synthesize while still retaining rich functionality. PAs can combine the functionality and flexibility of peptides; since there are 20 natural amino acids, there are practically numerous $\left(>10^{9}\right)$ possible sequences, even for short (18mers) peptides such as the one studied in this work. Naturally, the design of IDP based hydrophilic domains can be inspired by biology, utilizing the immense pre-existing knowledge base of proteins. To date, only few examples demonstrated exciting functionality using disordered domain in PA. ${ }^{17,19,20,33,34}$

Here, we study the self-assembly and encapsulation capabilities of two IDPAs, composed of hydrophilic domain inspired by the neurofilament-low disordered tail domain and a dendritic branching unit used for conjugating the lipophilic tails. ${ }^{28,35,36}$ Using turbidity, Small-angle X-ray Scattering (SAXS) and cryogenic transmission electron microscopy (cryo-TEM) measurements, we found that the IDPAs self-assembled into well-defined, lowdispersity nanoparticles. In addition, we show that the IDPAs' sensitivity to $\mathrm{pH}$ leads to a tunable and robust organization of the self-assembled nanoparticles. We further show that minor alterations in the peptide sequence can lead to alteration in IDPA-IDPA interaction and the macroscopic arrangement. Last, we demonstrate the potential of using the $\mathrm{pH}$ induced shape transition as a release mechanism for the design of nano carriers.

\section{Results and discussion}

\section{Synthesis and the structure of IDPAs}

IDP sequences were synthesized on an automated solid-phase peptide synthesizer using Fmoc-protected amino acids. The hydrophilic peptide domain sequence is an 18 mer aminoacid polyampholyte, inspired by the intrinsically disordered carboxy domain of the protein neurofilament-low (NF-L). ${ }^{28,29,36,37}$ Once the IDP sequences synthesis was completed, an aromatic branching unit containing two allyl or propargyl functionalities and a carboxylic acid was used to cap the N-terminus of the IDP sequence. After the branching units were 
a)
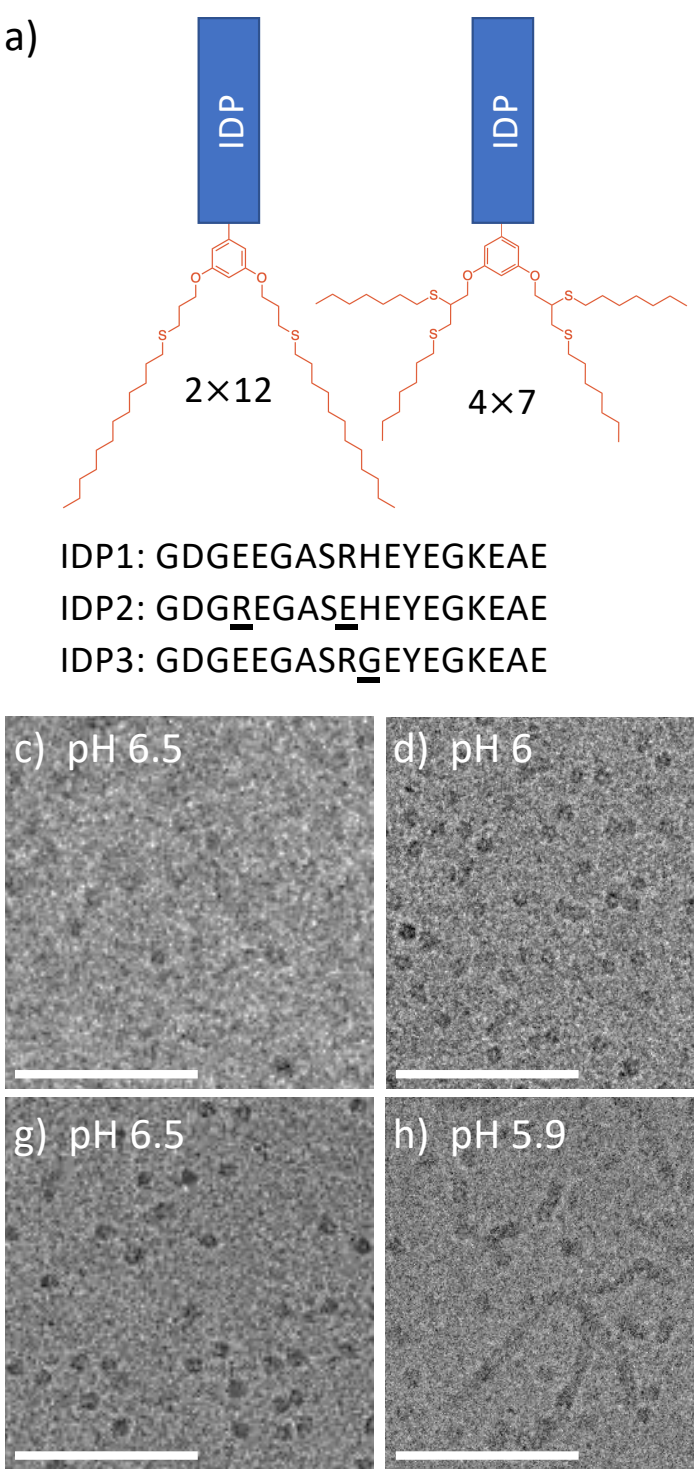
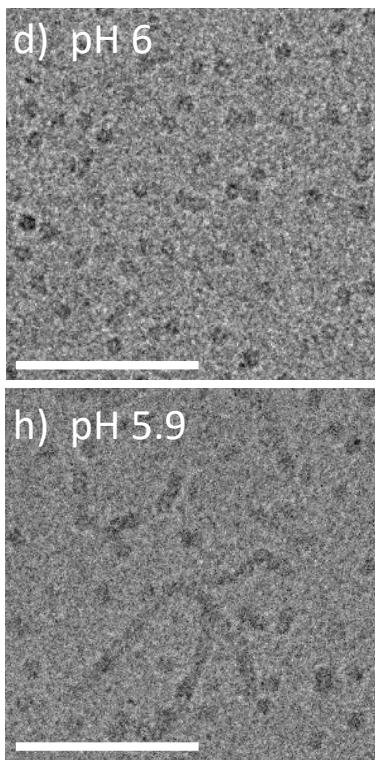
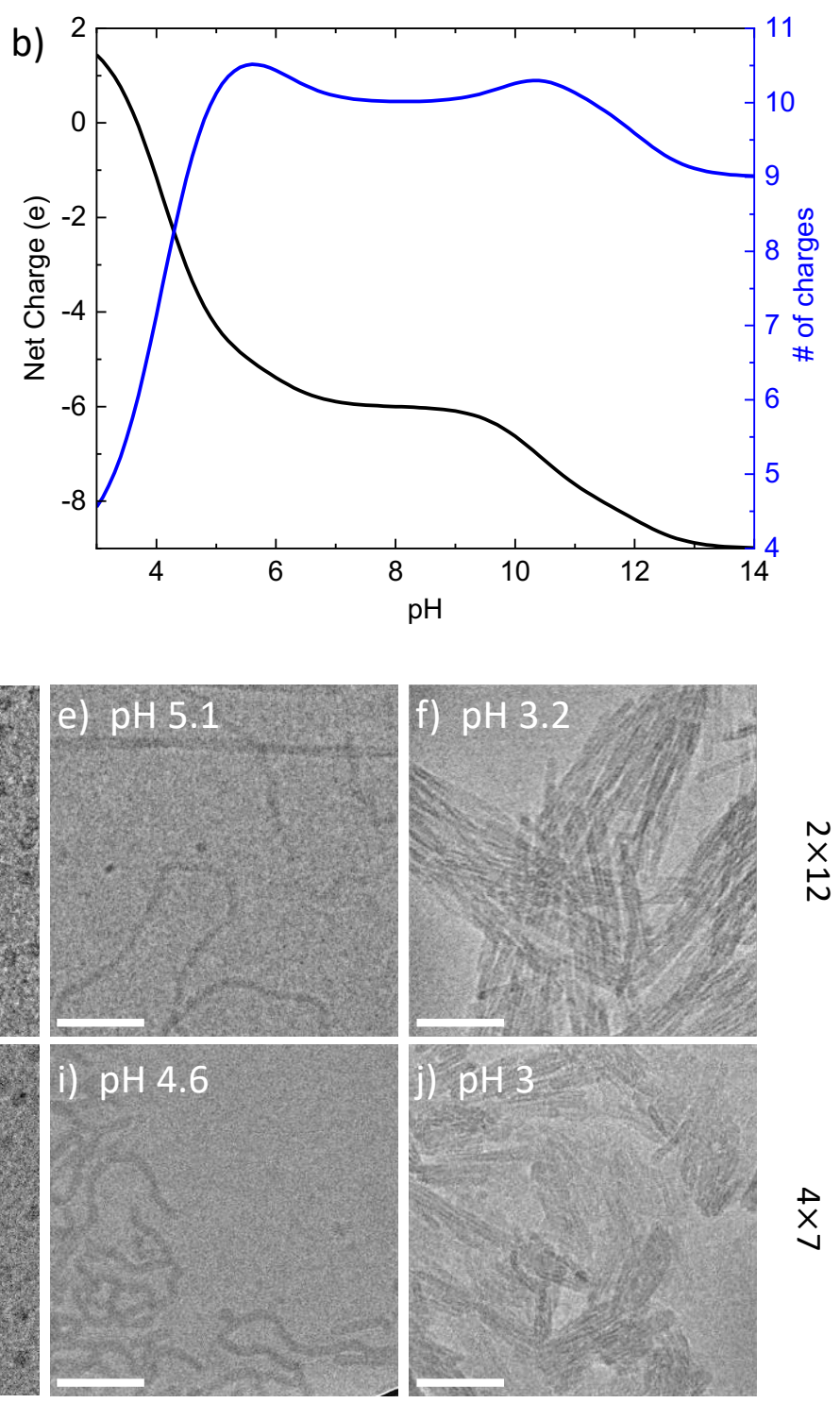

$\stackrel{\sim}{\underset{N}{N}}$

$\stackrel{\leftrightarrow}{\times}$

Figure 1: (a) Schematics of the IDPAs with two tail variants $(2 \times 12$ and $4 \times 7)$ and the one-letter IDP's sequences used in this study. (b) Net charge (black) and the number of charged amino acids (blue) of IDPA1 hydrophilic domain as a function of $\mathrm{pH}$. (c-j) IDPA1 Cryo-TEM images showing of self-assembly of (c-f) $2 \times 12$ and (g-j) $4 \times 7$ at various marked pHs. Cryo-TEM images show (c,d,g) spherical micelles at low $\mathrm{pH},(\mathrm{e}, \mathrm{h}, \mathrm{i})$ coexistence with worm-like micelles at intermediate $\mathrm{pH}$, and (f,j) aggregated micellar rods at low $\mathrm{pH}$. Scale bar is $100 \mathrm{~nm}$ 
conjugated, the capped peptides were cleaved from the resin using TFA, and hydrophobic end-groups containing thiols (dodecane-thiol and heptane-thiol) were conjugated to the allyl or propargyl moieties through thiol-ene or -yne click reactions, respectively. ${ }^{38-41}$ We term the tail-group variants by $2 \times 12$ and $4 \times 7$, to represent the number and length of the alkyl chains and the amphiphiles as IDPA1 (Fig. 1a). The one letter amino acid sequence of IDPA1 is GDGEEGASRHEYEGKEAE.

Notably, the peptide's sequence includes 11 protonatable residues, allowing for the net charge of the peptide to vary significantly as a function of $\mathrm{pH}$ (Fig. 1b). Specifically, at approximately $\mathrm{pH} 5.5$ there is a decrease in the net charge and in the number of charged residues due to Aspartic Acid and Glutamic Acid residues' protonation. The critical micelle concentrations (CMC) of $11 \mu \mathrm{M}$ were determined using the solvatochromic dye Nile Red (Supplementary Figure S1). A CMC of $5 \mu \mathrm{M}$ is in the typical micro-molar range for peptide amphiphiles. ${ }^{21,42-44}$

The peptide's degree of disorder was experimentally verified by measuring the circular dichroism (CD) spectrum of samples of the peptide (unconjugated) and the two IDPA1 variants (Supplementary Figure S2). In addition, the peptide sequence displays a high probability for disorder and the absence of regular secondary structure using the NetSurfP2.0 bioinformatic algorithm (Supplementary Figure S3). ${ }^{45}$

\section{Micellar nanostructures at high $\mathrm{pH}$}

We expected IDPA1 to show little to no sensitivity at high $\mathrm{pH}$ where the peptides' net charge state remains constant (Fig. 1b). Indeed, above $\mathrm{pH} \sim 6$, we find that both tailvariants assemble into nanoscopic spherical micelles, which were visualized via cryo-TEM (Fig. 1c-j). At this slightly acidic $\mathrm{pH}$, the micelles showed repulsion and remained miscible at a relatively high IDPA concentration $(10 \mathrm{mg} / \mathrm{ml})$. SAXS revealed low-dispersity spherical nanostructures, typical for structured particles. A core-shell form-factor was used to fit the SAXS data and provided the radius of the hydrophobic core $R_{\text {core }}=1.25 \pm 0.09 \mathrm{~nm}$, the width 
of the peptide shell (hydrophilic domain region surrounding the core) $w_{\text {shell }}=2.12 \pm 0.05$ $\mathrm{nm}$, and the respective average electron densities, $\rho_{\text {core }}=284 \mathrm{e} / \mathrm{nm}^{3}$ and $\rho_{\text {shell }}=355 \mathrm{e} / \mathrm{nm}^{3}$ (Fig. 2b). Using the SAXS fit and the values from Harpaz et al., ${ }^{46}$ we estimate the number of monomers for $4 \times 7$ and $2 \times 12$ IDPA1 at pH 7.5 to be approximately 13 and 40, respectively.

The SAXS measurements further revealed the composite dimensionality of the spherical micelles. Using the Kratky analysis, ${ }^{47}$ we found a bell-shaped curve at lower $q$, which corresponds to the 3D nature of the micelle at larger length-scales. However, the linear increase at larger $q$ suggests an unfolded state of the peptides at smaller length-scales (Fig. 2c). Approaching $\mathrm{pH} \sim 6$, we find a mild alteration in the SAXS signal, indicating a structural rearrangement at lower $\mathrm{pH}$ that we discuss next.

\section{pH induced phase-transition}

The peptide sequence is a strong polyampholyte; hence, the self-assembly of IDPA1 is expected to depend on $\mathrm{pH}$. Therefore, lowering the $\mathrm{pH}$ towards the $\mathrm{pI}$ can facilitate a structural phase-transition that depends on the IDP charge density and the amphiphiles' electrostatic interaction. Turbidity measurements performed on both IDPAs revealed a clear difference in sample translucency above and below pH 6 (Fig. 2a). This transition indicates a macromolecular aggregation of the self-assembled structures at low $\mathrm{pH}$. Below $\mathrm{pH}$, the nanostructures interact to produce larger and more ordered aggregates. Figure 2a showcases the transition from translucent to opaque solutions. The turbidity of both variants changes abruptly at $\mathrm{pH} \sim 6$ and 4.5 , for the $4 \times 7$ and $2 \times 12$, respectively.

Cryo-TEM micrographs of IDPA1 variants in the $\mathrm{pH}$ range 3-6.5 confirm the microscopic transition hinted at by the turbidity measurements (Fig. 1c-j). The micrographs show a transition from spherical micelles at $\mathrm{pH} 6.5$ to elongated worm-like micelles lower than

$\mathrm{pH}$ 5.5. Furthermore, at lower $\mathrm{pHs}$ the worm-like micelles are strongly interacting and aggregating. In the case of $4 \times 7$, the image taken at $\mathrm{pH} 5.9$ nicely shows the coexistence of spherical and worm-like micelles (Fig. 1h). In addition, the sphere-to-rod transition and 
micellar growth is earlier for the $4 \times 7$ variant and is consistent with its higher hydrophobicity and turbidity measurements.
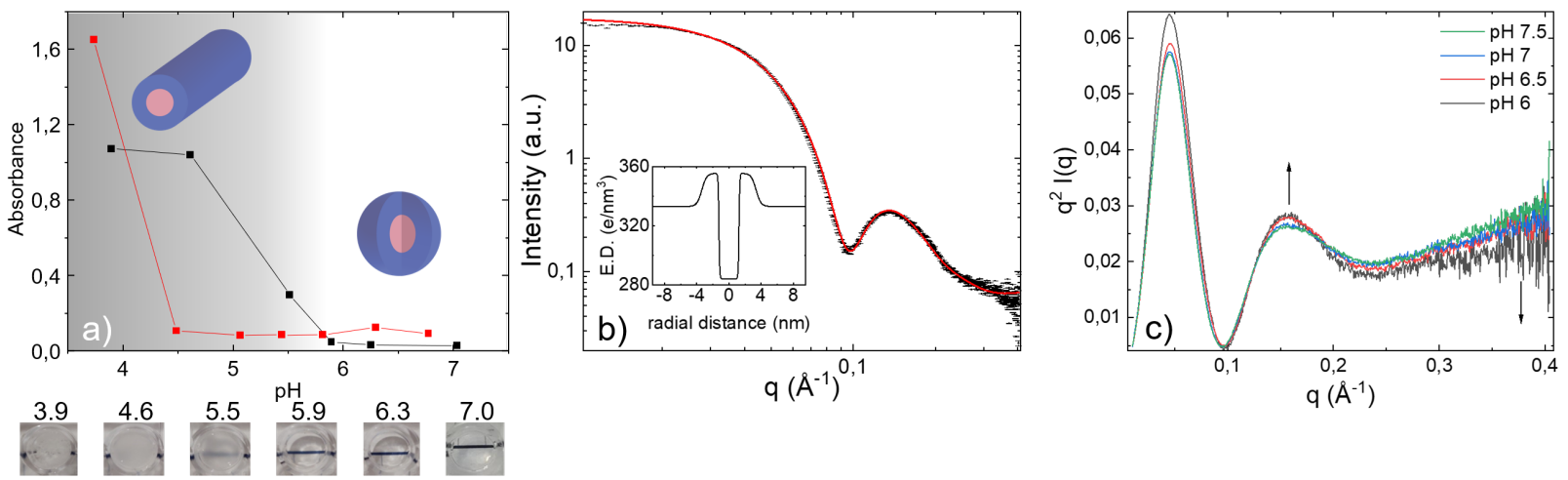

Figure 2: (a) Turbidity measurement of $4 \times 7$ (black) and $2 \times 12$ (red) IDPA1 with schematic representation of phase transition from spherical to cylindrical micelles. Results show an increase in turbidity when lowering the $\mathrm{pH}$, indicating a transition into large assemblies. Below: photographs of the $4 \times 7$ IDPA1 samples measured in the experiment (numbers above photos indicate the $\mathrm{pH}$ ). b) Spherical core-shell form-factor fit for the SAXS data. Inset, electron density profile used in the fit. c) Kratky analysis with a bell-shaped curve at lower $q$, corresponding to 3D nature of the micelle at larger length-scales, and linear increase at larger $q$ resulted from the unfolded state of the peptides at smaller length-scales.

Further verification of the phase transition is clearly shown using SAXS (Figs. 3a,b). At low $\mathrm{pH}$, the scattering is qualitatively different and is no longer a sum of independent spherical scatterers producing a form-factor SAXS signal. Instead, the SAXS signal now includes an additional structure-factor signal produced by the inter-particles' correlation. The structure-factor peaks position match a $2 \mathrm{D}$ hexagonal lattice for $2 \times 12$ and a $1 \mathrm{D}$ lattice for $4 \times 7$ with the corresponding unit-cell spacing of $d_{H}=10.2 \mathrm{~nm}$ and $d_{L}=9 \mathrm{~nm}$. The highly-dense packing is also evident bythe cryo-TEM micrographs showing organization of the micellar rods at low pHs (Fig. 1f,j).

\section{Engineering the self-assembly by point mutation}

The added value of using peptides as the hydrophilic domain is the possibility to tune the interactions via small alterations in the sequence, such as a single mutation. We recently demonstrated that a similar sequence peptide alters its self-interaction via a single point 
mutation. ${ }^{48}$ Short-ranged transient interactions are also present in the original neurofilament disordered protein, ${ }^{28,29,49-52}$ presumably due to the oppositely charged amino-acids along the polyampholytic intrinsically disordered C-terminus domain. We synthesized a sequence variant by changing the Glutamic Acid at position 4 with the Arginine at position 9, termed IDPA2. By doing so, we are conserving the net charge and the functional relation between $\mathrm{pH}$ and charge but altering the charge distribution at the hydrophilic peptide domain. Such alteration is expected to alter the ionic bridging between the IDPs. ${ }^{28,49,52}$

In addition, when considering the observed structural $\mathrm{pH}$ sensitivity, with a phasetransition being located somewhere between $\mathrm{pH} 4$ and 6 (Figs. 1c,d and 3), a possible origin for the transition can be the charging state of the Histidine amino-acid. To verify this hypothesis, we designed a slightly modified hydrophilic domain sequence, termed IDPA3, that replaces the Histidine at position 10 with a neutral and $\mathrm{pH}$ insensitive Glycine residue. Notably, the two new IDPA variants are still considered highly disordered and are assigned the random coil conformation by the predictors (Supplementary Figure S4).

Indeed, we found that the hydrophilic domain (i.e., the disordered peptide) and its interactions in the assembly control the complex aggregations at low pH. SAXS experiments show that replacing the Histidine with a Glycine served to strengthen the interaction between the worm-like micelles (Fig. 3d). However, slightly changing the sequence's order, namely the IDPA2 variant, noticeably changes the complex aggregation (Fig. 3c). For the $2 \times 12$ variant, we saw a significant weakening of micelle-micelle interactions in the worm-like phase, at 150 $\mathrm{mM}$ and $1 \mathrm{M}$ salt concentration, demonstrated by the diminished structure-factor scattering (Fig. 3a). However, the $4 \times 7$ variant showed a similar weakening of the interactions only at $1 \mathrm{M}$ salt pointing towards non-trivial electrostatic interaction ${ }^{27,28,52}$ between the IDPs (Fig. $3 b)$. 


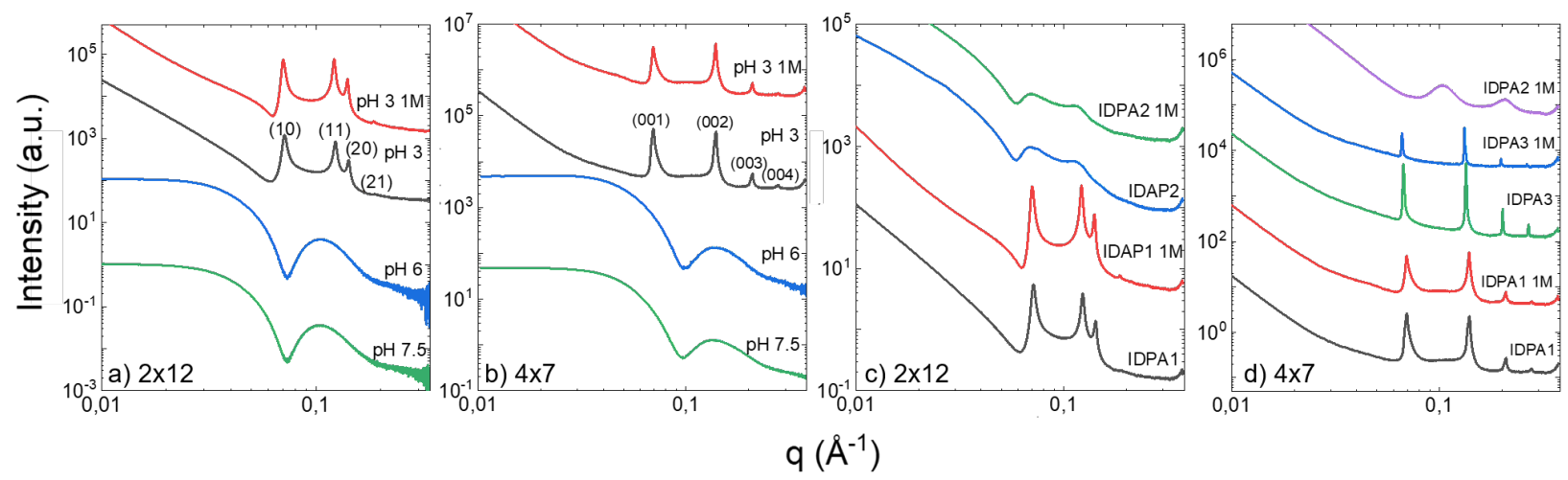

Figure 3: SAXS measurements of (a) $2 \times 12$ and (b) $4 \times 7$ IDPA1 variants at different pHs. Above $\mathrm{pH} 6$ the scattering profile pertains to spherical micelles. Below $\mathrm{pH} 6$, the scattering is dominated by a structure-factor. The $2 \times 12$ variant forms a hexagonal phase with a spacing of $d_{H}=10.2$, stable at $1 \mathrm{M} \mathrm{NaCl}$ (red line). For the $4 \times 7$ variant, the scattering at low pHs is dominated by a $1 \mathrm{D}$ phase structure with a spacing of $d_{L}=9 \mathrm{~nm}$.(c) Comparison of SAXS measurements of $2 \times 12$ for IDPA1 and IDPA2 at $\mathrm{pH} 3$, in either $150 \mathrm{mM}$ salt or $1 \mathrm{M}$ (labeled). The small change in charge distribution has a dramatic effect on the interaction of the worm-like micelles. The sharp structure-factor peaks are replaced with wide and shallow peaks, indicating weaker correlations. (d) Comparison of SAXS measurements of $4 \times 7$ variants at $\mathrm{pH} 3$, in either $150 \mathrm{mM}$ salt or $1 \mathrm{M}$ (labeled). The mesophase remains the same for IDPA1 and IDPA3, while the IDPA2 variant shows a pronounced weakening of the inter-micelle correlations. 


\section{Cargo encapsulation and release}

After the pH-depended self-assembly into micelles and worm-like micelles was characterized, we wanted to evaluate how the shape transition will affect encapsulated cargo release. We chose butyl ester of 7-(Diethylamino)coumarin-3-carboxylic acid as the hydrophobic cargo and used a dialysis set-up to study the hydrophobic dye's release from the assembled structures. Solutions containing micelles of $2 \times 12$ and $4 \times 7$ IDPAs $(\mathrm{pH} 6.5)$ were mixed with a stock solution of the dye, followed by filtration of the residual un-encapsulated dyes. Next, the samples' $\mathrm{pH}$ was adjusted to $\mathrm{pH} 4$ by adding a few $\mu \mathrm{L}$ of $\mathrm{HCl}$ to transform the spherical micellar assemblies into the worm-like micelles. As expected, while the micelle solution was clear, the worm-like micelle solution became highly turbid (Fig. 4).

Next, the two solutions were transferred into dialysis tubes and placed in buffer solutions ( $\mathrm{pH} 6.5$ or 4 for the micelles and worm-like micelles, respectively) containing bovine serum albumin (BSA). The BSA's role was to scavenge the released dyes and avoid their aggregation and dissolution due to their poor aqueous solubility. The solutions were then placed in a shaking incubator at $37^{\circ} \mathrm{C}$, and samples were taken periodically from the outer solution and characterized by a spectrophotometer to determine the released dye's concentration.

We find that the spherical micelles' release was faster than the release from the worm-like assemblies (Fig. 4). To quantify how the phase-transition affects the release, we continued measuring the release after the solutions' $\mathrm{pH}$ was adjusted from 6.5 to 4 or vice-versa, and the samples were placed in suitable fresh buffers. As controls, we used samples kept under the same initial $\mathrm{pH}$ and placed them into fresh buffers. It was fascinating to see the change in release rate due to the $\mathrm{pH}$-induced shape transition as worm-like micelles that were transformed into spherical micelles (solid blue lines in Fig. 4a,b) started to release the dyes faster, showing a similar rate as the non-altered micelle control (dashed red lines in Fig. 4a,b). Simultaneously, a slower release was observed for spherical micelles, which were transformed into worm-like micelles (solid red lines and dashed blue lines in Fig. 4a,b).

The changes in release rate due to the $\mathrm{pH}$-induced change in mesophases were observed 
for both $2 \times 12$ and $4 \times 7$ IDPAs, with the effect being more significant for the latter. To ensure that the release rates were genuinely affected by the change in the assemblies' shape, we prepared a non-responsive amphiphile with two dodecane alkyl chains by replacing the hydrophilic IDP with a PEG chain of similar molecular weight. The PEG-based amphiphile $($ PEG-2 $\times 12)$ self-assembled into micelles with a diameter of hydration of around $10 \mathrm{~nm}$ at both high and low pH (Supplementary Figure S5), demonstrating that its assembly is not responsive to $\mathrm{pH}$ at the tested range. We were encouraged to see that the control amphiphile release rates were not affected by the pH-jump after 3 hours. These results indicate that it is indeed the change in the shape of the IDPA assemblies due to altered interaction between the hydrophilic peptide domains, which affects the release rate.

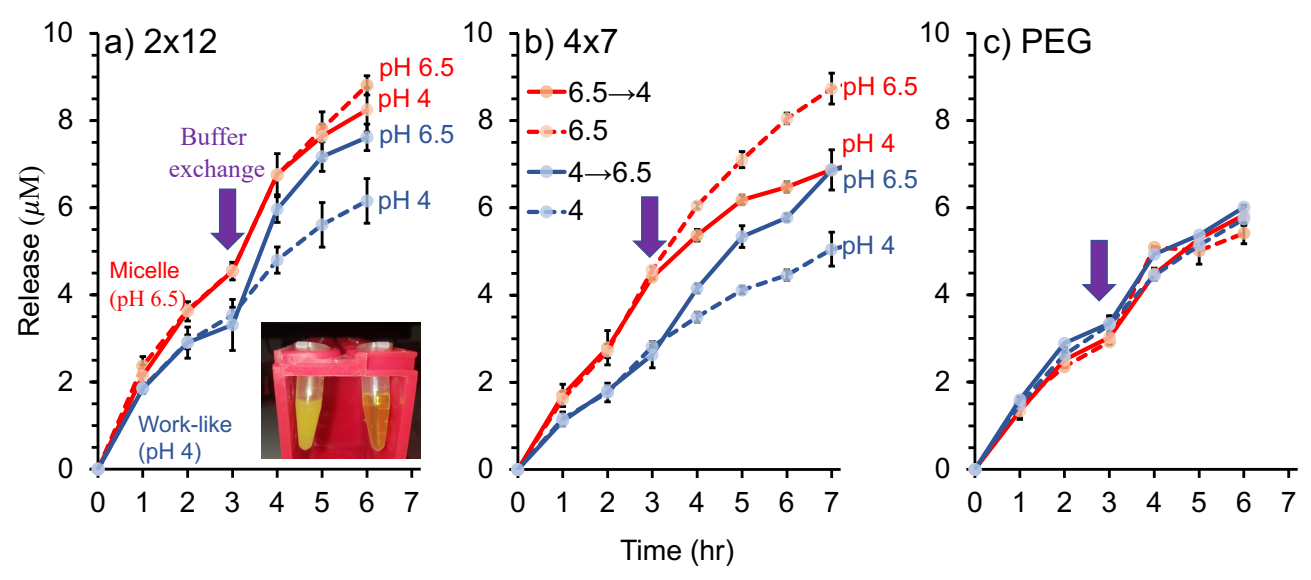

Figure 4: Encapsulation measurements. Accumulative released dye concentration for (a) $2 \times 12$, (b) $4 \times 7$ IDPAs and (c) PEG- $2 \times 12$. Blue and red data-points represent a baseline $\mathrm{pH}$ for the experiments of 6.5 and 4, respectively. After 3 hours the buffer was exchanged either to induce structural mesophase transition via $\mathrm{pH}$ trigger (solid lines), or to identical and fresh buffer (dashed line). PEG- $2 \times 12$ amphiphiles at $\mathrm{pH} 6.5$ and $\mathrm{pH} 4$ show no $\mathrm{pH}$ release trigger. Inset, representative photo of the encapsulated dye in (left) worm-like micelles at $\mathrm{pH} 4$ and (right) spherical micelles at $\mathrm{pH} 6.5$.

\section{Discussion}

We demonstrated the IDPA could serve as a valuable platform having stimuli-responsive selfassembly. Importantly, these nanoscopic assemblies are well-ordered, although originating 
from disordered peptides. When modeling the IDPA interactions and phase-transitions presented here, it is essential to understand whether the dominant interactions are non-specific, e.g., based on the total charge-density, or specific, e.g., conformational-based interactions. A generic model for a spherical-to-cylindrical micelle phase-transition will be agnostic to the charged peptide domain's details, aside from the charge distribution. ${ }^{53}$ Such a model, detailed for brevity in the supplementary information, describes the free-energy balance between the energy associated with the hydrophilic region's charge-density and the energetic price paid for inducing a curvature that deviates from the intrinsic one. Essentially, lowering the $\mathrm{pH}$ results in a lower net charge that allows the hydrophilic peptide to compress. Assuming that the intrinsic curvature favors cylindrical morphology, this can facilitate the decrease in bending energy with a denser hydrophilic region.

However, the mutated variants' experimental finding suggests that we must also consider the sequence's details and charges distribution along the peptide chain. Therefore, a sequence-dependent interaction term must be included in the free-energy calculation, describing the interaction between the amino-acids along two parallel and offset peptides ${ }^{28,36,52}$ (Supplementary Information).

The experimental results on the IDPA2 variant show that indeed such a small change significantly affects the inter-micelle interactions, and as a result, the aggregation phase was dramatically changed. Therefore, the minute alteration between the original sequence and its variants suggests sequence-specific interactions. Moreover, the entire IDPA architecture, including the hydrophobic domain, also plays a role in the pH-response and the macroscopic aggregated mesophase (i.e., at low $\mathrm{pH}$ ). For the $4 \times 7$ hydrophobic domain, there is a clear structural difference between the IDPA2 and the other variants at high salt concentration. For the $2 \times 12$ hydrophobic domain, while the condensed phase structural correlation weakens for the IDPA2 variants, they are salt-independent between 0.15-1 M. Indeed, as suggested by our theoretical modeling, the IDPA3 condensed phase (at $\mathrm{pH}=3$ ) is somewhat similar to the original variant (see supplementary materials). 


\section{Conclusions}

We investigated the conjugation of disordered polypeptide domains with hydrocarbon dendrimers into IDPA. We find that the interactions between the peptides lead to tunable self-assembled nanostructures. The IDPA hydrophilic domains are weakly interacting and disordered by nature, generally associated with transient behavior. Nonetheless, the IDPA self-assembly is remarkably forming ordered nanoparticles.

Moreover, the IDPA system shows $\mathrm{pH}$ response and structural phase-transition. It can be useful as a trigger for cargo release, similar to already demonstrated amphiphilic polymerbased nanoparticle systems with drug release enzymatic response. ${ }^{54}$

We further demonstrated that minute alteration in the disordered peptide sequence and dendrimer architecture could significantly impact the nanoscopic and macroscopic lengthscales. This platform also enables in-situ modifications of the IDPs and the study of the mutual interaction between IDPs. Last, as we showed here, modification of the IDP domain, and its interaction with the surrounding, plays a critical role in the IDPA's self-assembly and structural transformation from external cues. We expect that this platform can be furthered explored for targeted drug-delivery where tailored biological signals will induce phase-transition and expedite release.

\section{Materials and methods}

\section{Synthesis and purification}

All peptides were synthesized at the Blavatnik Center for Drug Discovery (BCDD) at Tel Aviv University using automated Fmoc solid-phase peptide synthesis using The Liberty Blue $^{T M}$ automated microwave peptide synthesizer (CEM, Matthews, NC, USA). After the coupling of the last amino acid, either 3,5-bis(allyloxy)benzoic acid or 3,5-bis(prop-2-yn-1-

yloxy)benzoic $\operatorname{acid}^{55}$ were coupled to the N-termius of the peptide. The capped peptides 
were cleaved from the resin usign standard conditions (95\% trifluoroacetic acid (TFA) (v/v), $2.5 \% \mathrm{H} 2 \mathrm{O}(\mathrm{v} / \mathrm{v})$, and $2.5 \%$ triisopropylsilane (v/v) for 3 hours). The cleaved di-allyl or dipropargyl were purified by Waters AutoPurification system ${ }^{T M}$ (MS directed LC) and were further reacted in thiol-ene or thiol-yne reactions with 1-dodecanethiol or 1-heptanethiol, respectively, as described below to yield IDPAs $2 \times 12$ and $4 \times 7$, respectively.

IDPA1-diallyl: Exact Mass 2164.88. Detected Mass: ES mode +: $723.28[(\mathrm{M}+3 \mathrm{H}) / 3]$ and $1084.21[(\mathrm{M}+2 \mathrm{H}) / 2]$.

IDPA1-2 $2 \times 12: 54 \mathrm{mg}(0.025 \mathrm{mmol})$ of peptide 2 was dissolved with $350 \mu \mathrm{l}$ of phosphate buffer (pH 7.4,100mM) using gentle hitting, then $1 \mathrm{ml}$ of DMF was added. $5.1 \mathrm{mg}$ of DMPA (0.02 mmol) was separately solubilized with $100 \mu \mathrm{l}$ of DMF and added to the peptide solution flowed by addition of $239.5 \mu \mathrm{l}$ 1-dodecanethiol $(0.997 \mathrm{mmol})$. The solution was purged with $\mathrm{N}_{2}$ for 15 minutes and then stirred under UV light for 2 hours. Next, the crud mixture was placed in dialysis membrane with MWCO of 3,000 Da and dialyzed against DI water for $12 \mathrm{hr}$. The solution was lyophilized and final purification was performed using preparativescale reversed-phase HPLC (Waters AutoPurification system). The product was confirmed by LC/MS. ACN was removed by rotary evaporation and the solution was further lyophilized yielding a white solid product $31.26 \mathrm{mg}$ (48\% yield). Exact mass 2569.23. Detected mass: ES mode +: $857.98[(\mathrm{M}+3 \mathrm{H}) / 3]$ and $1286.40[(\mathrm{M}+2 \mathrm{H}) / 2]$.

IDPA1-dipropargyl: Exact Mass 2160.85. Detected mass: ES mode +: 721.91 [(M+3H)/3] and $1082.23[(\mathrm{M}+2 \mathrm{H}) / 2]$.

IDPA1-4 $\times 7: 58 \mathrm{mg}(0.027 \mathrm{mmol})$ of peptide 1 were dissolved with $350 \mu \mathrm{l}$ of phosphate

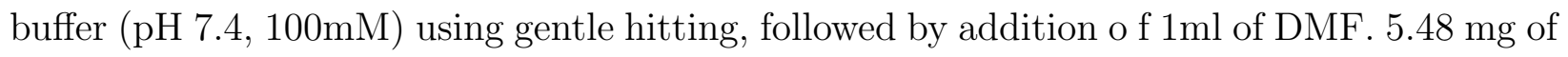
2,2-Dimethoxy-2-phenylacetophenone (DMPA, $0.021 \mathrm{mmol}$ ) was separately solubilized with $100 \mu \mathrm{l}$ of DMF and added to the peptide solution flowed by addition of $338 \mu \mathrm{l}$ 1-heptanethiol (2.1 mmol). The solution was purged with $\mathrm{N}_{2}$ for 15 minutes and then stirred under UV light for 2 hours. Next, the crud mixture was placed in dialysis membrane with MWCO of 3,000 Da and dialyzed against DI water for $12 \mathrm{hr}$. The solution was lyophilized and the 
product was purified using reparative-scale reversed-phase HPLC (Waters AutoPurification system). The product was confirmed by LC/MS. ACN was removed by rotary evaporation and further lyophilized yielding a white solid product $19.2 \mathrm{mg}$ (26\% yield). Exact Mass 2689.24. Detected Mass: ES mode +: $898.17[(\mathrm{M}+3 \mathrm{H}) / 3]$ and $1346.72[(\mathrm{M}+2 \mathrm{H}) / 2]$.

IDPA2-diallyl: Exact Mass 2164.88. Detected Mass: ES mode +: $723.26[(\mathrm{M}+3 \mathrm{H}) / 3]$ and $1084.19[(\mathrm{M}+2 \mathrm{H}) / 2]$.

IDPA2-2 $\times 12$ : Was synthesized similarly to IDPA1. $51 \mathrm{mg}(0.024 \mathrm{mmol})$ of IDP2- were mixed with $4.8 \mathrm{mg}$ of DMPA and $226 \mu \mathrm{l}$ of 1-dodecanethiol and reacted and purified as was described for the synthesis of IDPA1 to yield $30.7 \mathrm{mg}$ (50\% yield). MS analysis: Exact Mass: 2569.23 Detected Mass: ES mode +: $858.17[(\mathrm{M}+3 \mathrm{H}) / 3]$ and $1286.78[(\mathrm{M}+2 \mathrm{H}) / 2]$.

IDPA2-dipropargyl: Exact Mass 2160.85. Detected Mass: ES mode +: $721.89[(\mathrm{M}+3 \mathrm{H}) / 3]$ and $1082.14[(\mathrm{M}+2 \mathrm{H}) / 2]$.

IDPA2-4 $\times 7$ : Was synthesized and purified similarly to IDPA1. $54 \mathrm{mg}(0.025 \mathrm{mmol})$ of IDP2-dipropargyl were mixed with $5.1 \mathrm{mg}$ of DMPA and $315 \mu \mathrm{l}$ of 1-heptanethiol and reacted and purified as was described for the synthesis of IDPA1 to yield $26.8 \mathrm{mg}$ (40\% yield). MS analysis: Exact Mass: 2689.24 Detected Mass: ES mode +: $1346.78[(\mathrm{M}+2 \mathrm{H}) / 2]$ and 898.22 $[(\mathrm{M}+3 \mathrm{H}) / 3]$.

IDPA3-dipropargyl: Exact Mass 2080.81. Detected Mass: ES mode +: $695.06[(\mathrm{M}+3 \mathrm{H}) / 3]$ and $1041.95[(\mathrm{M}+2 \mathrm{H}) / 2]$.

IDPA3-4×7: Was synthesized and purified similarly to IDPA1. $14 \mathrm{mg}(0.0067 \mathrm{mmol})$ of IDP3-dipropargyl were mixed with $1.36 \mathrm{mg}$ of DMPA and $84 \mu \mathrm{l}$ of 1-heptanethiol, and reacted and purified as was described for the synthesis of IDPA1 to yield $9 \mathrm{mg}$ (51\%). MS analysis: Exact Mass: 2609.20 Detected Mass: ES mode +: 1306.83 [(M+2H)/2] and 871.47 $[(\mathrm{M}+3 \mathrm{H}) / 3]$.

PEG-Dendron $(2 \times 12)$ synthesis: Dendron: $600 \mathrm{mg}(2.5 \mathrm{mmol})$ of 3,5-(allyloxy) benzoic acid, ${ }^{55} 3.11 \mathrm{~g}$ of 1-dodecanethiol $(15 \mathrm{mmol})$ and $38.4 \mathrm{mg}$ of 2,2-Dimethoxy-2- phenylacetophenone (DMPA; $0.15 \mathrm{mmol}$ ) were dissolved in $800 \mu$ l of DMF. The solution was purged 
with N2 for 15 minutes and then stirred under UV light for 2 hours. Next, crud mixture was loaded on silica column, thiol excess was washed with 10:90 ethyl acetate and hexane (v/v) and $2 \times 12$ dendron compound was eluted with 30:70 ethyl acetate and hexane (v/v), The fractions that contained the product were unified,evaporated and dried under high vacuum obtaining 1.5 g yellowish oily compound (92\% yield). 1H NMR (400 MHz, Chloroform-d) $\delta 7.23(\mathrm{~d}, J=2.2 \mathrm{~Hz}, 2 \mathrm{H}), 6.69(\mathrm{t}, J=2.3 \mathrm{~Hz}, 1 \mathrm{H}), 4.10(\mathrm{t}, J=6.1 \mathrm{~Hz}, 4 \mathrm{H}), 2.70(\mathrm{t}$, $J=7.1 \mathrm{~Hz}, 4 \mathrm{H}), 2.52(\mathrm{t}, J=7.0 \mathrm{~Hz}, 4 \mathrm{H}), 2.07(\mathrm{p}, J=6.5 \mathrm{~Hz}, 4 \mathrm{H}), 1.59(\mathrm{p}, J=7.3$ $\mathrm{Hz}, 4 \mathrm{H}), 1.26-1.38(\mathrm{~m}, 37 \mathrm{H}), 0.88$ (t, $J=6.8 \mathrm{~Hz}, 6 \mathrm{H})$. PEG-Dendron $(2 \times 12)$ : $70 \mathrm{mg}$ of $2 \mathrm{kDa}$ PEG-amine ${ }^{56}$ were dissolved in $100 \mu \mathrm{l}$ of DCM and $67.3 \mathrm{mg}$ of $2 \times 12$ dendron, and $40 \mathrm{mg}$ of (2-(1H-benzotriazol-1-yl)-1,1,3,3-tetramethyluronium hexafluorophosphate HBTU were dissolved in DCM:DMF 1:1 (1mL) followed by addition of DIPEA $60 \mu$ l were added to the PEG-amine solution and allowed to stir for 3 hour at room temperature. The crude mixture was loaded on a MeOH based LH20 SEC column. The fractions that contained the product were unified and the $\mathrm{MeOH}$ was evaporated in vacuum to obtain $76 \mathrm{mg}$ (82\%) of the PEG based amphiphile. 1H NMR (400 MHz, Chloroform-d) $\delta 6.91$ (d, J=2.2 Hz, 2H), $6.69(\mathrm{t}, J=5.8 \mathrm{~Hz}, 1 \mathrm{H}), 6.57(\mathrm{t}, J=2.2 \mathrm{~Hz}, 1 \mathrm{H}), 4.08(\mathrm{t}, J=6.1 \mathrm{~Hz}, 4 \mathrm{H}), 3.83-3.45(\mathrm{PEG}$ backbone), $3.38(\mathrm{~s}, 3 \mathrm{H}), 2.77(\mathrm{t}, J=6.5 \mathrm{~Hz}, 2 \mathrm{H}), 2.70-2.63(\mathrm{~m}, 6 \mathrm{H}), 2.52(\mathrm{t}, J=7.4 \mathrm{~Hz}$, 4H), $2.05(\mathrm{p}, J=6.6 \mathrm{~Hz}, \mathrm{H}), 1.88(\mathrm{p}, J=6.6 \mathrm{~Hz}, 2 \mathrm{H}), 1.62-1.52(\mathrm{~m}, 4 \mathrm{H}), 1.45-1.26(\mathrm{~m}$, $40 \mathrm{H}), 0.88(\mathrm{t}, J=6.7 \mathrm{~Hz}, 6 \mathrm{H})$.

\section{SAXS and cryo-TEM sample preparation}

The IDPA or peptide powder was first fluidized in purified water (Milli-Q) at twice the desired concentration. The solution was then titrated with $\mathrm{NaOH}$ to a $\mathrm{pH}$ where the solution became more homogeneous (preferably a $\mathrm{pH}$ where the IDPAs are soluble in water). Titration was monitored using a pH probe (Sentek P13 pH Electrode). Following titration, $50 \mu l$ of the solution was combined with $50 \mu \mathrm{l}$ of $2 \mathrm{X}$ buffer of choice to achieve a $\mathrm{pH}$ in the vicinity to the desired one. The $2 \mathrm{X}$ buffers Acetic Acid (pH 3-4.5), MES pH (5-6.5), and MOPS (pH 
7-7.5) were prepared at $200 \mathrm{mM}$ to achieve final buffer molarity of $100 \mathrm{mM}$ after mixing with IDPA or peptide solution 1:1 (vol:vol).

\section{SAXS}

For solubilizing conditions (above the transition $\mathrm{pH}$, generally above $\mathrm{pH} 6$ ), samples were measured at three synchrotron facilities: Beamline B21, Diamond Light Source, UK, beamline 12.3.1, SIBYLS, Advanced Light Source, Berkeley, USA and beamline SWING, SOLEIL synchrotron facility, Paris, France.

For phase-separating samples that display sediment (below the transition $\mathrm{pH}$, generally pH 3-5.5), measurements were performed using an in-house X-ray scattering system, with a Genix3D (Xenocs) low divergence $\mathrm{Cu} \mathrm{K} \mathrm{K}_{\alpha}$ radiation source (wavelength of $\lambda=1.54 \AA$ ) with a Pilatus 300K (Dectris) detector and scatterless slits setup, ${ }^{57}$ as well as beamline I22 at Diamond Light Source. Here, samples were measured inside $1.5 \mathrm{~mm}$ quartz capillaries (Hilgenberg).

\section{Peptide's SAXS analysis}

The unconjugated peptide by itself was measured using SAXS at different $\mathrm{pH}$ levels to test the effect on its ensemble-averaged structure. The peptide at each $\mathrm{pH}$ was measured at four different concentrations to extrapolate to the non-interacting "zero-concentration" peptides scatterings. From the low momentum transfer $(q)$ regime of the extrapolated zeroconcentration scattering curves, we extracted the radius of gyration $\left(R_{g}\right)$ and the forward scattering, $I(0)$, using the Guinier analysis (Supplementary Figure S6). When examining the values of $R_{g}$ from high to low $\mathrm{pH}$, it seems to remain a constant until $\mathrm{pH} \sim 5.5$ and gradually increase below it. However, this increase in size can be explained by a simultaneous increase in the effective mass of the peptides (increase in forwarding scattering) due to a decrease in inter-peptide repulsion near the isoelectric point $(\mathrm{pI})$. 


\section{Cryo-TEM}

Cryo-TEM specimen preparation was performed by applying a $6 \mu l$ drop of the studied solution to a perforated carbon film supported on a 200-mesh TEM copper grid, thinning (blotting), and removing excess solution. Next, the sample was vitrified in liquid ethane at its freezing point $\left(-183^{\circ} \mathrm{C}\right)$. The procedure was carried out at a controlled temperature $\left(25^{\circ} \mathrm{C}\right)$ and water saturation. The vitrified specimens were stored under liquid nitrogen $\left(-196{ }^{\circ} \mathrm{C}\right)$ until examination. The samples were then examined using a Tecnai T12 G2 (FEI, The Netherlands) TEM operated at an accelerating voltage of $120 \mathrm{kV}$, keeping specimen temperature below $-170{ }^{\circ} \mathrm{C}$ during transfer and observation. Images were digitally recorded on a Gatan Ultrascan 1000 cooled CCD camera using the Gatan Digital Micrograph software

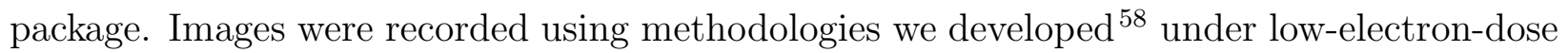
conditions to minimize electron beam radiation damage.

\section{Turbidity}

All measurements were recorded on a TECAN Infinite M200Pro device. The amphiphiles were treated and prepared in the same manner as previously described to achieve a final concentration of $5 \mathrm{mg}$ ml-1. $100 \mu \mathrm{l}$ of each solution was loaded onto a 96 wells plate. The absorbance at $600 \mathrm{~nm}$ was scanned for each well.

\section{CD}

Circular dichroism (CD) measurements were performed using a commercial CD spectrometer (Applied Photophysics Chirascan). Both IDPAs, $2 \times 12$ and $4 \times 7$, and the unconjugated IDP, were placed in a glass cuvette with a $10 \mathrm{~mm}$ path length. The IDPAs and peptide were mixed with a phosphate buffer to achieve a concentration of 0.05 and $0.1 \mathrm{mg} \mathrm{ml}-1$, respectively. Measurements were performed using phosphate buffer since the buffers used for the X-ray scattering experiments (mainly MOPS and MES) have high absorbance in the relevant CD 
wavelengths. The 190-260 nm wavelength range was probed in $1 \mathrm{~nm}$ steps, with 0.5 secs at each point. Three measurements were performed for each and averaged.

\section{CMC}

The amphiphile was dissolved in the diluent (15ml MOPS buffer solution ( $\mathrm{pH} 7.4$ ), $7.5 \mu \mathrm{L}$ of Nile red stock solution $(2.5 \mathrm{mM}$ in Ethanol) were added and mixed to give a final concentration of $1.25 \mu \mathrm{M})$ to give the final concentration of $400 \mu \mathrm{M}$ IDPA and then sonicated for 5 minutes. This solution was repeatedly diluted by a factor of 1.5 with diluent. $100 \mu \mathrm{L}$ of each solution were loaded onto a 96 wells plate. The fluorescence emission intensity was scanned for each well (550 nm Emission intensity scan: 580-800 nm) using TECAN Infinite M200Pro plate reader. Maximum emission intensity was plotted vs. concentration in order to determine the CMC. This procedure was repeated thrice.

\section{Cargo release experiment}

Release experiments of hydrophobic dye (butyl-coumarin) were performed using a dialysis tube (Mini GeBAflex-Kit, 8 kDa MWCO), volume range $10-250 \mu \mathrm{L}$. A volume of $80 \mu \mathrm{L}$ of the micelles and worm-like micelle solutions were placed in the dialysis tubes, and each tube was immersed into $8 \mathrm{ml}$ buffer at $\mathrm{pH} 6.5$ or 4 with $0.5 \mathrm{mg} / \mathrm{ml}$ of BSA, respectively.

\section{Acknowledgement}

We thank Diamond Light Source for time on Beamlines B21 (SM24693) and I22 (SM21971), Advanced Light Source for time on Beamline SIBYLS 12.3.1 (SB-00941), and the SOLEIL synchrotron facility for time on Beamline SWING (20170798). The work was supported by the Israel Science Foundation (grant numbers 1454/20, 1553/18, 3292/19, 1117/16), Deutsche Forschungsgemeinschaft through SFB 958, NWU-TAU, the LMU-TAU collaboration initiatives, and DFG project GR1030/14-1. We thank Dr. Elvira Haimov and Dr. 
Boris Redko from the Blavatnik Center for Drug Discovery for their help with the synthesis and purification of the IDPAs. We also acknowledge fruitful discussions with Nathan Gianneschi, Joachim Rädler, Ram Avinery, and technical support of Mingming Zhang.

\section{Supporting Information Available}

The Supporting Information is available free of charge at XXX.

\section{References}

(1) Kulkarni, C. V. Lipid crystallization: from self-assembly to hierarchical and biological ordering. Nanoscale 2012, 4, 5779-91.

(2) Dengler, E. C.; Liu, J.; Kerwin, A.; Torres, S.; Olcott, C. M.; Bowman, B. N.; Armijo, L.; Gentry, K.; Wilkerson, J.; Wallace, J., et al. Mesoporous silica-supported lipid bilayers (protocells) for DNA cargo delivery to the spinal cord. Journal of Controlled Release 2013, 168, 209-224.

(3) Rodriguez, N.; Pincet, F.; Cribier, S. Giant vesicles formed by gentle hydration and electroformation: A comparison by fluorescence microscopy. Colloids and Surfaces B: Biointerfaces 2005, 42, 125-130.

(4) Gunatillake, P. A.; Adhikari, R. Biodegradable synthetic polymers for tissue engineering. Eur Cell Mater 2003, 5, 1-16.

(5) Segal, M.; Ozery, L.; Slor, G.; Wagle, S. S.; Ehm, T.; Beck, R.; Amir, R. J. Architectural Change of the Shell-Forming Block from Linear to V-Shaped Accelerates Micellar Disassembly, but Slows the Complete Enzymatic Degradation of the Amphiphiles. Biomacromolecules 2020, 21, 4076-4086. 
(6) Harnoy, A. J.; Rosenbaum, I.; Tirosh, E.; Ebenstein, Y.; Shaharabani, R.; Beck, R.; Amir, R. J. Enzyme-responsive amphiphilic PEG-dendron hybrids and their assembly into smart micellar nanocarriers. Journal of the American Chemical Society 2014, 136, $7531-7534$

(7) Peer, D.; Karp, J. M.; Hong, S.; Farokhzad, O. C.; Margalit, R.; Langer, R. Nanocarriers as an emerging platform for cancer therapy. Nature Nanotechnology 2007, 2, 751-760.

(8) Mai, Y.; Eisenberg, A. Self-assembly of block copolymers. Chemical Society Reviews 2012, 41, 5969.

(9) Carlsen, A.; Lecommandoux, S. Self-assembly of polypeptide-based block copolymer amphiphiles. Current Opinion in Colloid and Interface Science 2009, 14, 329-339.

(10) Zhuang, J.; Gordon, M. R.; Ventura, J.; Li, L.; Thayumanavan, S. Multi-stimuli responsive macromolecules and their assemblies. Chemical Society reviews 2013, 42, 7421-35.

(11) Miyata, K.; Christie, R. J.; Kataoka, K. Polymeric micelles for nano-scale drug delivery. Reactive and Functional Polymers 2011, 71, 227-234.

(12) Segal, M.; Avinery, R.; Buzhor, M.; Shaharabani, R.; Harnoy, A. J.; Tirosh, E.; Beck, R.; Amir, R. J. Molecular precision and enzymatic degradation: From readily to undegradable polymeric micelles by minor structural changes. Journal of the American Chemical Society 2017, 139, 803-810.

(13) Uversky, V. N. The mysterious unfoldome: structureless, underappreciated, yet vital part of any given proteome. BioMed Research International 2009, 2010.

(14) He, B.; Wang, K.; Liu, Y.; Xue, B.; Uversky, V. N.; Dunker, A. K. Predicting intrinsic disorder in proteins: an overview. Cell research 2009, 19, 929-949. 
(15) Uversky, V. N. Intrinsically disordered proteins in overcrowded milieu: Membraneless organelles, phase separation, and intrinsic disorder. Current opinion in structural biology 2017, 44, 18-30.

(16) Hartgerink, J. D.; Beniash, E.; Stupp, S. I. Self-assembly and mineralization of peptideamphiphile nanofibers. Science 2001, 294, 1684-1688.

(17) Chin, S. M.; Synatschke, C. V.; Liu, S.; Nap, R. J.; Sather, N. A.; Wang, Q.; Álvarez, Z.; Edelbrock, A. N.; Fyrner, T.; Palmer, L. C.; Szleifer, I.; Olvera De La Cruz, M.; Stupp, S. I. Covalent-supramolecular hybrid polymers as muscle-inspired anisotropic actuators. Nature Communications 2018, 9, 1-11.

(18) Hartgerink, J. D.; Beniash, E.; Stupp, S. I. Peptide-amphiphile nanofibers: A versatile scaffold for the preparation of self-assembling materials. Proceedings of the National Academy of Sciences 2002, 99, 5133-5138.

(19) Silva, G. A.; Czeisler, C.; Niece, K. L.; Beniash, E.; Harrington, D. A.; Kessler, J. A.; Stupp, S. I. Selective Differentiation of Neural Progenitor Cells by High-Epitope Density Nanofibers. Science 2004, 303, 1352-1355.

(20) Peters, D.; Kastantin, M.; Kotamraju, V. R.; Karmali, P. P.; Gujraty, K.; Tirrell, M.; Ruoslahti, E. Targeting atherosclerosis by using modular, multifunctional micelles. Proceedings of the National Academy of Sciences 2009, 106, 9815-9819.

(21) Acar, H.; Samaeekia, R.; Schnorenberg, M. R.; Sasmal, D. K.; Huang, J.; Tirrell, M. V.; LaBelle, J. L. Cathepsin-Mediated Cleavage of Peptides from Peptide Amphiphiles Leads to Enhanced Intracellular Peptide Accumulation. Bioconjugate Chemistry 2017, 28, 2316-2326.

(22) Bull, S. R.; Guler, M. O.; Bras, R. E.; Meade, T. J.; Stupp, S. I. Self-assembled peptide amphiphile nanofibers conjugated to MRI contrast agents. Nano Letters 2005, 5, 1-4. 
(23) Tovar, J. D.; Claussen, R. C.; Stupp, S. I. Probing the interior of peptide amphiphile supramolecular aggregates. Journal of the American Chemical Society 2005, 127, 73377345.

(24) Paramonov, S. E.; Jun, H. W.; Hartgerink, J. D. Self-assembly of peptide-amphiphile nanofibers: The roles of hydrogen bonding and amphiphilic packing. Journal of the American Chemical Society 2006, 128, 7291-7298.

(25) Trent, A.; Marullo, R.; Lin, B.; Black, M.; Tirrell, M. Structural properties of soluble peptide amphiphile micelles. Soft Matter 2011, 7, 9572-9582.

(26) Oates, M. E.; Romero, P.; Ishida, T.; Ghalwash, M.; Mizianty, M. J.; Xue, B.; Dosztányi, Z.; Uversky, V. N.; Obradovic, Z.; Kurgan, L.; Dunker, A. K.; Gough, J. D2P2: Database of disordered protein predictions. Nucleic Acids Research 2013, 41 , $508-516$.

(27) Kornreich, M.; Avinery, R.; Malka-Gibor, E.; Laser-Azogui, A.; Beck, R. Order and disorder in intermediate filament proteins. FEBS Letters 2015, 589, 2464-2476.

(28) Kornreich, M.; Malka-Gibor, E.; Zuker, B.; Laser-Azogui, A.; Beck, R. Neurofilaments Function as Shock Absorbers: Compression Response Arising from Disordered Proteins. Physical Review Letters 2016, 117, 148101.

(29) Malka-Gibor, E.; Kornreich, M.; Laser-Azogui, A.; Doron, O.; Zingerman-Koladko, I.; Harapin, J.; Medalia, O.; Beck, R. Phosphorylation-Induced Mechanical Regulation of Intrinsically Disordered Neurofilament Proteins. Biophysical Journal 2017, 112, 892900.

(30) DeForte, S.; Uversky, V. N. Order, disorder, and everything in between. Molecules 2016, 21, 1090 . 
(31) Wei, M. T.; Elbaum-Garfinkle, S.; Holehouse, A. S.; Chen, C. C. H.; Feric, M.; Arnold, C. B.; Priestley, R. D.; Pappu, R. V.; Brangwynne, C. P. Phase behaviour of disordered proteins underlying low density and high permeability of liquid organelles. Nature Chemistry 2017, 9, 1118-1125.

(32) Shin, Y.; Brangwynne, C. P. Liquid phase condensation in cell physiology and disease. Science 2017, 35\%, eaaf4382.

(33) Dzuricky, M.; Rogers, B. A.; Shahid, A.; Cremer, P. S.; Chilkoti, A. De novo engineering of intracellular condensates using artificial disordered proteins. Nature Chemistry 2020, 12, 814-825.

(34) Mozhdehi, D.; Luginbuhl, K. M.; Simon, J. R.; Dzuricky, M.; Berger, R.; Varol, H. S.; Huang, F. C.; Buehne, K. L.; Mayne, N. R.; Weitzhandler, I., et al. Genetically encoded lipid-polypeptide hybrid biomaterials that exhibit temperature-triggered hierarchical self-assembly. Nature chemistry 2018, 10, 496-505.

(35) Uversky, V. N.; Dunker, A. K. Understanding protein non-folding. Biochimica et Biophysica Acta - Proteins and Proteomics 2010, 1804, 1231-1264.

(36) Kornreich, M.; Malka-Gibor, E.; Laser-Azogui, A.; Doron, O.; Herrmann, H.; Beck, R. Composite bottlebrush mechanics: $\alpha$-internexin fine-tunes neurofilament network properties. Soft Matter 2015, 11, 5839-5849.

(37) Laser-Azogui, A.; Kornreich, M.; Malka-Gibor, E.; Beck, R. Neurofilament assembly and function during neuronal development. Current Opinion in Cell Biology 2015, 32, $92-101$.

(38) Hoyle, C. E.; Bowman, C. N. Thiol-ene click chemistry. Angewandte Chemie International Edition 2010, 49, 1540-1573. 
(39) Kade, M. J.; Burke, D. J.; Hawker, C. J. The power of thiol-ene chemistry. Journal of Polymer Science Part A: Polymer Chemistry 2010, 48, 743-750.

(40) Konkolewicz, D.; Gray-Weale, A.; Perrier, S. Hyperbranched polymers by thiol- yne chemistry: from small molecules to functional polymers. Journal of the American Chemical Society 2009, 131, 18075-18077.

(41) Lowe, A. B. Thiol-yne 'click'/coupling chemistry and recent applications in polymer and materials synthesis and modification. Polymer 2014, 55, 5517-5549.

(42) Vincenzi, M.; Accardo, A.; Costantini, S.; Scala, S.; Portella, L.; Trotta, A.; Ronga, L.; Guillon, J.; Leone, M.; Colonna, G.; Rossi, F.; Tesauro, D. Intrinsically disordered amphiphilic peptides as potential targets in drug delivery vehicles. Molecular BioSystems 2015, 11, 2925-2932.

(43) Klass, S. H.; Smith, M. J.; Fiala, T. A.; Lee, J. P.; Omole, A. O.; Han, B. G.; Downing, K. H.; Kumar, S.; Francis, M. B. Self-Assembling Micelles Based on an Intrinsically Disordered Protein Domain. Journal of the American Chemical Society 2019, 141, 4291-4299.

(44) Black, M.; Trent, A.; Kostenko, Y.; Lee, J. S.; Olive, C.; Tirrell, M. Self-assembled peptide amphiphile micelles containing a cytotoxic T-cell epitope promote a protective immune response in vivo. Advanced Materials 2012, 24, 3845-3849.

(45) Klausen, M. S.; Jespersen, M. C.; Nielsen, H.; Jensen, K. K.; Jurtz, V. I.; Sønderby, C. K.; Sommer, M. O. A.; Winther, O.; Nielsen, M.; Petersen, B.; Marcatili, P. NetSurfP-2.0: Improved prediction of protein structural features by integrated deep learning. Proteins: Structure, Function, and Bioinformatics 2019, 87, 520-527.

(46) Harpaz, Y.; Gerstein, M.; Chothia, C. Volume changes on protein folding. Structure 1994, 2, 641-649. 
(47) Orthaber, D.; Bergmann, A.; Glatter, O. SAXS experiments on absolute scale with Kratky systems using water as a secondary standard. Journal of Applied Crystallography 2000, 33, 218-225.

(48) Chakraborty, I.; Rahamim, G.; Avinery, R.; Roichman, Y.; Beck, R. Nanoparticle Mobility over a Surface as a Probe for Weak Transient Disordered Peptide-Peptide Interactions. Nano Letters 2019, 19, 6524-6534.

(49) Kornreich, M.; Malka-Gibor, E.; Laser-Azogui, A.; Doron, O.; Herrmann, H.; Beck, R. Composite bottlebrush mechanics: $\alpha$-internexin fine-tunes neurofilament network properties. Soft Matter 2015, 11, 5839-5849.

(50) Pregent, S.; Lichtenstein, A.; Avinery, R.; Laser-Azogui, A.; Patolsky, F.; Beck, R. Probing the interactions of intrinsically disordered proteins using nanoparticle tags. Nano Letters 2015, 15, 3080-3087.

(51) Morgan, I. L.; Avinery, R.; Rahamim, G.; Beck, R.; Saleh, O. A. Glassy Dynamics and Memory Effects in an Intrinsically Disordered Protein Construct. Physical Review Letters 2020, 125, 058001.

(52) Beck, R.; Deek, J.; Jones, J. B.; Safinya, C. R. Gel-expanded to gel-condensed transition in neurofilament networks revealed by direct forcemeasurements. Nature Materials 2010, 9, 40-46.

(53) Lerche, D.; Kozlov, M. M.; Markin, V. S. Electrostatic free energy and spontaneous curvature of spherical charged layered membrane. Biorheology 1987, 24, 23-34.

(54) Daniel, K. B.; Callmann, C. E.; Gianneschi, N. C.; Cohen, S. M. Dual-responsive nanoparticles release cargo upon exposure to matrix metalloproteinase and reactive oxygen species. Chemical Communications 2016, 52, 2126-2128. 
(55) Harnoy, A. J.; Papo, N.; Slor, G.; Amir, R. J. Mixing End Groups in Thiol-Ene/Yne Reactions as a Simple Approach toward Multienzyme-Responsive Polymeric Amphiphiles. Synlett 2018, 29, 2582-2587.

(56) Harnoy, A. J.; Rosenbaum, I.; Tirosh, E.; Ebenstein, Y.; Shaharabani, R.; Beck, R.; Amir, R. J. Enzyme-responsive amphiphilic PEG-dendron hybrids and their assembly into smart micellar nanocarriers. Journal of the American Chemical Society 2014, 136, $7531-7534$.

(57) Li, Y.; Beck, R.; Huang, T.; Choi, M. C.; Divinagracia, M. Scatterless hybrid metalsingle-crystal slit for small-angle X-ray scattering and high-resolution X-ray diffraction. Journal of Applied Crystallography 2008, 41, 1134-1139.

(58) Danino, D. Cryo-TEM of soft molecular assemblies. Current Opinion in Colloid and Interface Science 2012, 17, 316-329. 
Graphical TOC Entry

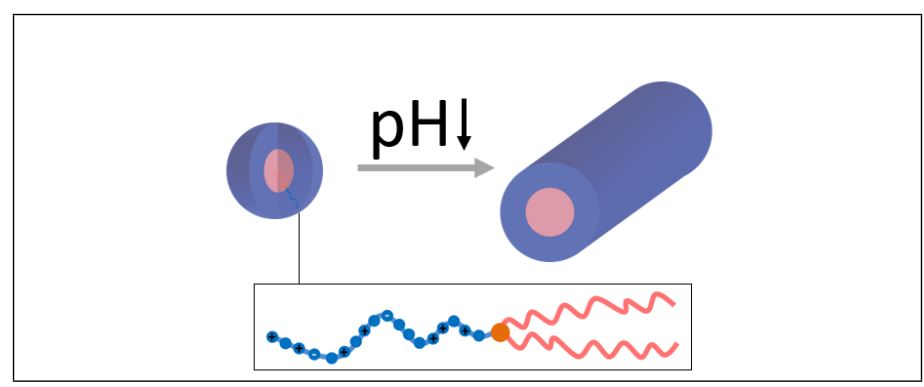

